Supporting Information For:

\title{
Size-Dependent PbS QD Surface Chemistry Investigated via Gel Permeation Chromatography
}

Adam Roberge, $\$$ John H. Dunlap, ${ }^{+}$Fiaz Ahmed,, Andrew B. Greytak ${ }^{*} *$

Fepartment of Chemistry and Biochemistry, University of South Carolina 29208, United States

\section{Corresponding Author}

*E-mail: greytak@mailbox.sc.edu

$\underline{\text { Contents }}$

$\underline{\text { Page }}$

Additional Methods. Reaction scheme for anhydrous lead oleate

S2

Figure S1. Absorbance spectra of PbS QDs before and after GPC purification

S3

Figure S2. Representative Thermogravimetric analysis of GPC-purified PbS QDs

S3

Figure S3. FTIR spectra of PbS QDs from normal and anhydrous syntheses

S4

Figure S4. ${ }^{1} \mathrm{H}$ NMR of large PbS QDs after purification showing two oleate species $\quad$ S4

Figure S5. Diffusion ordered NMR spectroscopy of PbS QDs, $\mathrm{Pb}(\mathrm{OA})_{2}$, and oleic acid $\quad \mathrm{S} 5$

Figure S6. Diffusion ordered NMR spectroscopy of $\mathrm{PbS} \mathrm{QDs}, \mathrm{Pb}(\mathrm{OA})_{2}$, and oleic acid $\quad \mathrm{S} 6$

Figure S7. Absorbance spectra of PbS QDs before GPC, after GPC, and after VT- NMR S7

Figure S8. ${ }^{1} \mathrm{H}$ NMR of $\mathrm{PbS}$ QDs synthesized using anhydrous $\mathrm{Pb}(\mathrm{OA})_{2}$ 
Methods: Reaction scheme for anhydrous lead oleate using procedure from Hendricks et al. ${ }^{1}$

Reaction 1:
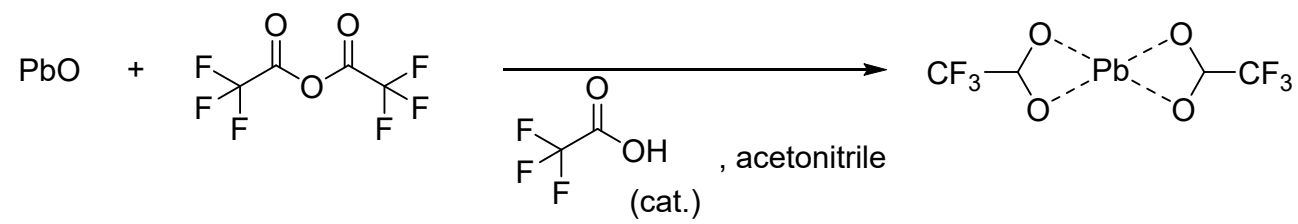

Reaction 2:

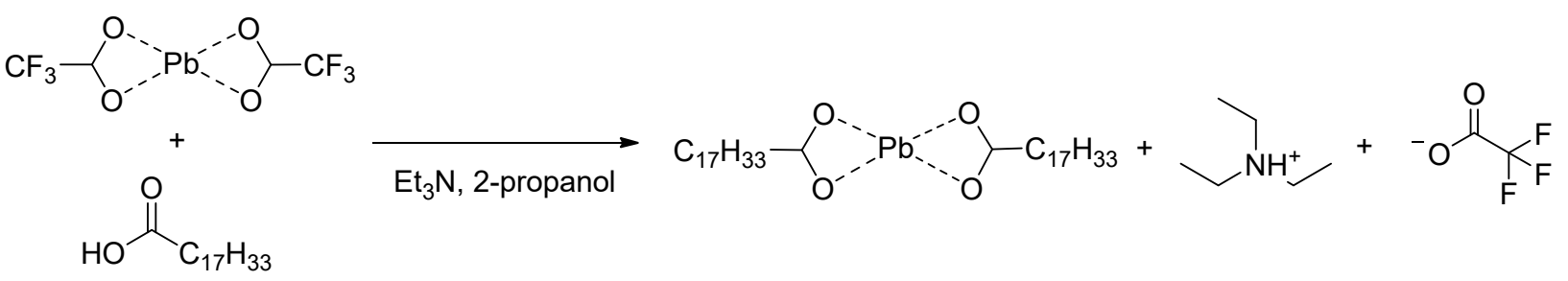

(1) Hendricks, M. P.; Campos, M. P.; Cleveland, G. T.; Plante, I. J.-L.; Owen, J. S. A Tunable Library of Substituted Thiourea Precursors to Metal Sulfide Nanocrystals. Science 2015, 348 (6240), 1226-1230. 
Figure S1.

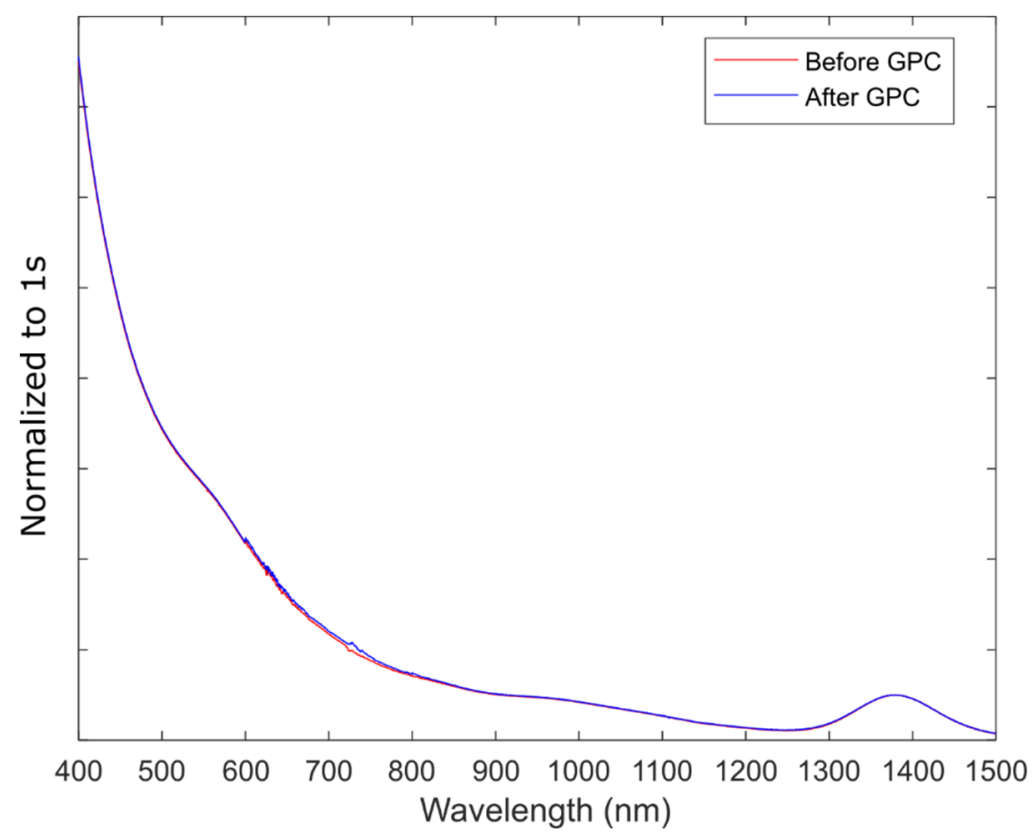

Absorbance spectra of PbS QDs before and after GPC purification, normalized according to the maximum absorbance value in the lowest-energy excited state peak.

Figure S2.

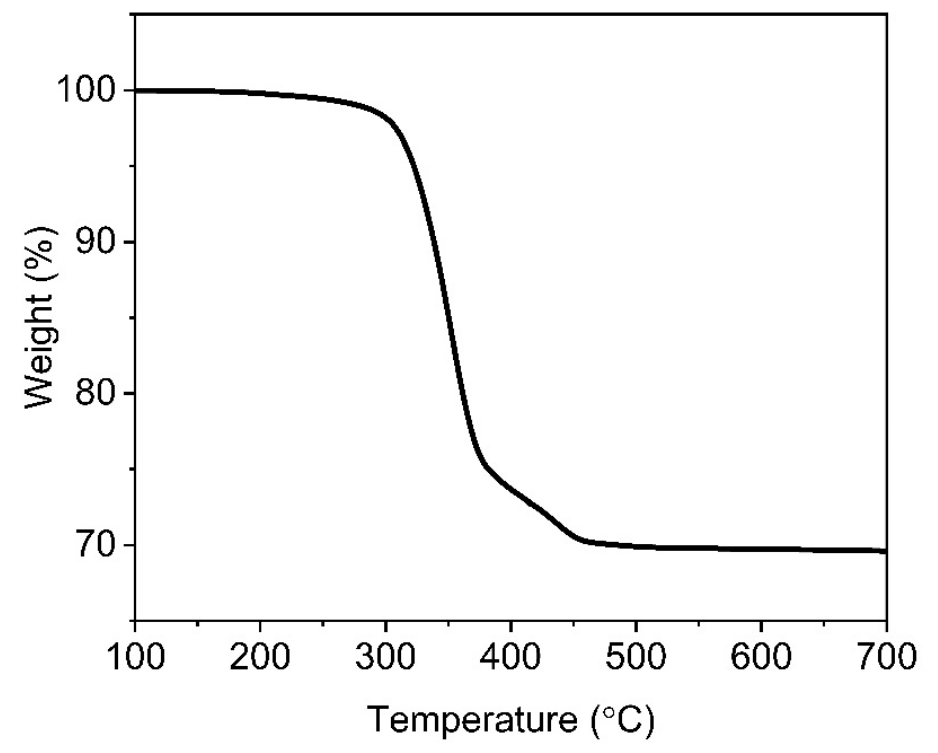

Thermogravimetric analysis of a representative GPC-purified PbS QD sample. A sample of GPC-purified QDs with nominal diameter $4.37 \mathrm{~nm}$ (1250 nm 1s peak) showed $231 \%$ mass loss on heating, similar to the oleate weight percentage of $32.9 \%$ determined by an NMR measurement on the same sample. 
Figure S3.

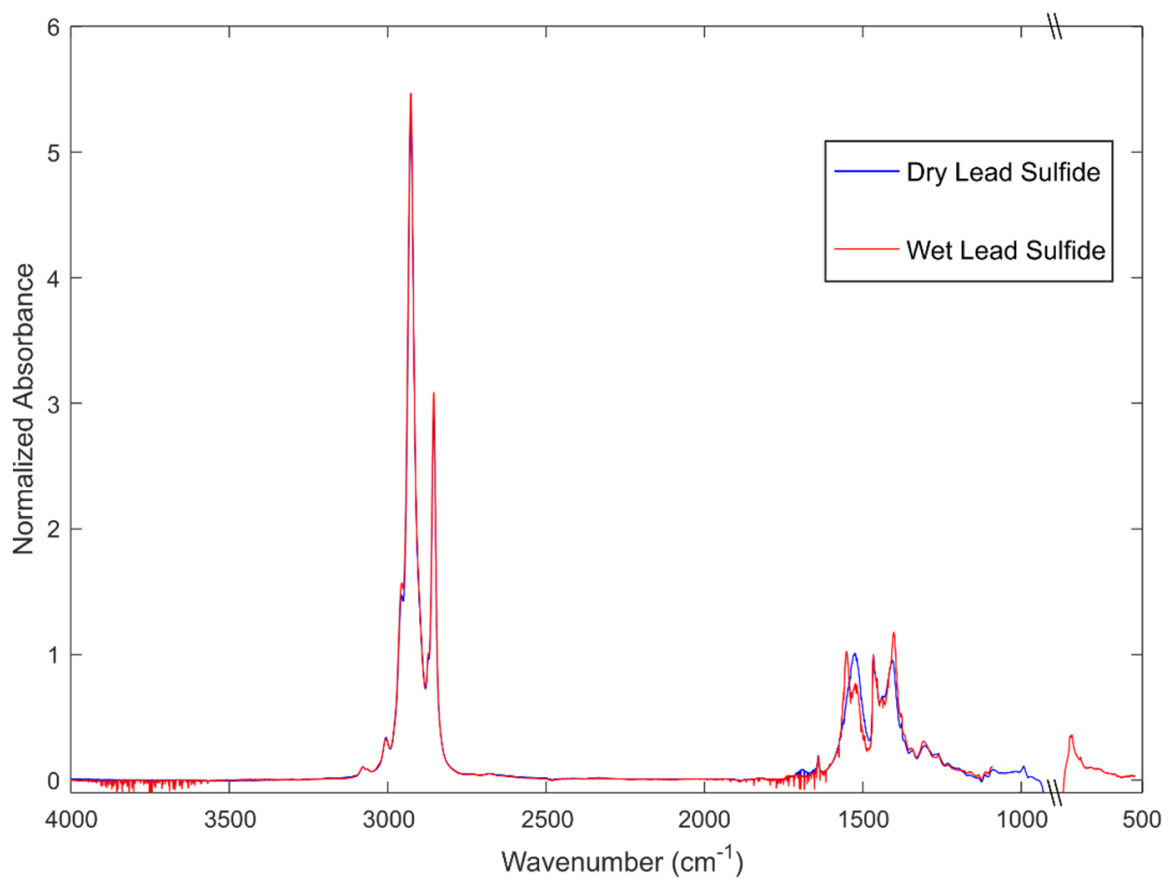

FTIR spectra of PbS QDs after GPC purification (in anhydrous tetrachloroethylene solution, with $\mathrm{KBr}$ plates) for normal and anhydrous synthetic methods.

Figure S4.

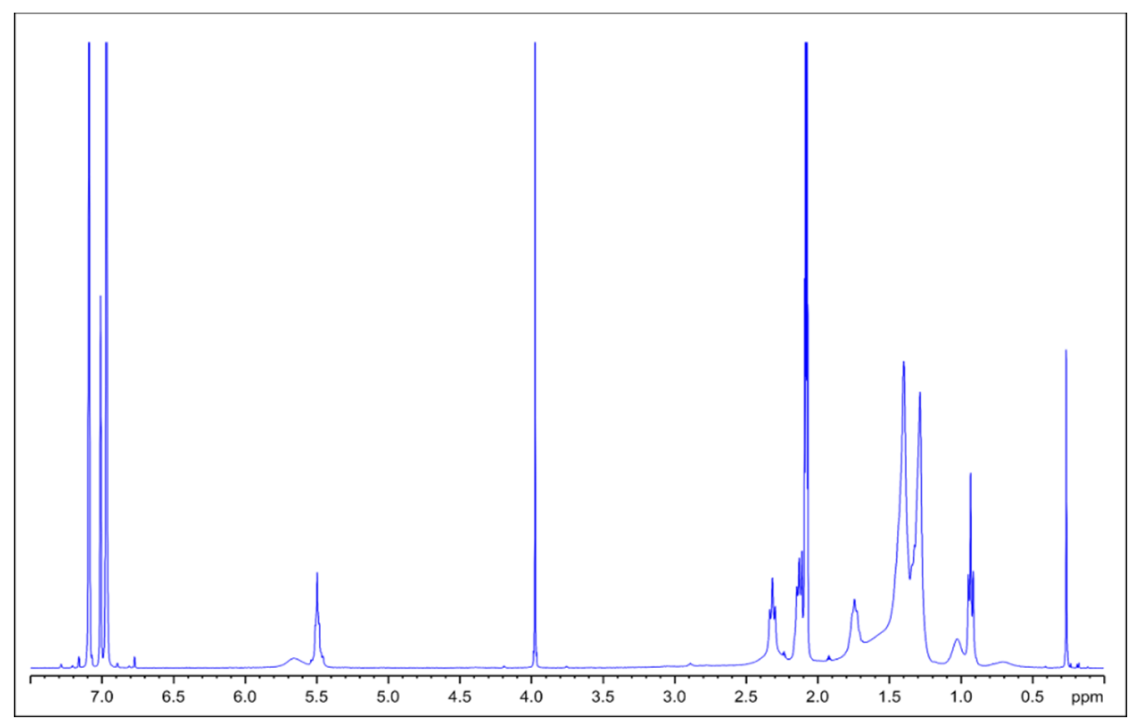

${ }^{1} \mathrm{H}$ NMR of large $(6.12 \mathrm{~nm}) \mathrm{PbS}$ QDs after purification showing two oleate species. 
Figure S5.

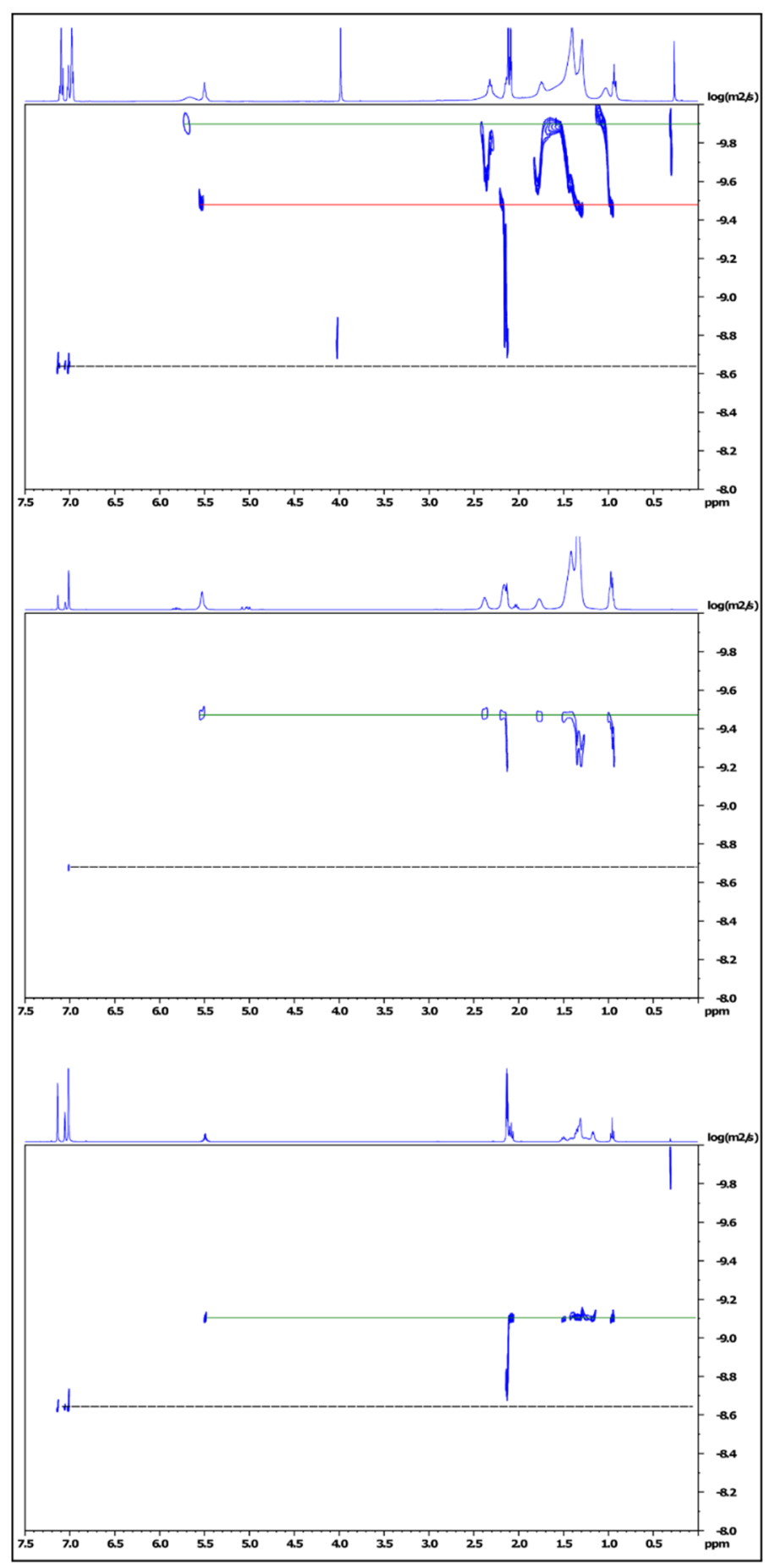

Diffusion ordered NMR spectroscopy (DOSY) of large-diameter PbS QDs (top). The diffusion constant $D$ for bound (broader) and free (sharper) olefin resonances were 5.5\% and 14.3\% of the toluene solvent, respectively. Middle: Independently prepared $\mathrm{Pb}(\mathrm{OA})_{2}$ in toluene $(15.6 \%$ of solvent $D$ ). Bottom: Oleic acid in toluene (35.0\% of solvent D). 
Figure S6.
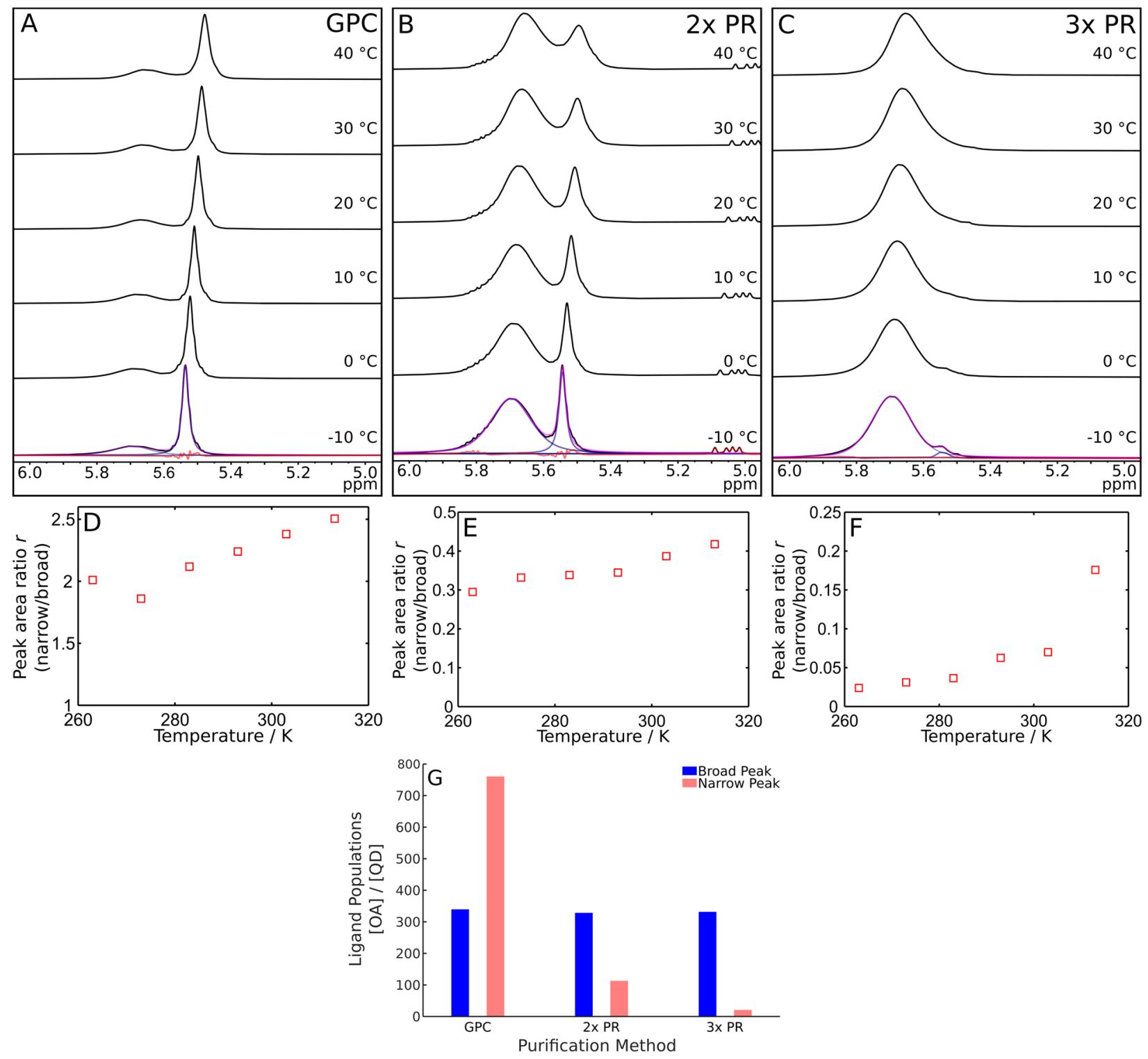

Variable-temperature NMR and ligand population quantification of similarly-sized large $\mathrm{PbS}$ QDs $(d \sim 5.9 \mathrm{~nm})$ with different purifications. (A-C) VT-NMR detail of PbS QDs with GPC, $2 \mathrm{x}$ PR, and 3x PR purifications, respectively. Representative Gaussian and Lorentzian fits of olefin peak areas for each sample at $-10{ }^{\circ} \mathrm{C}$ are provided. (D-F) Ratio $r$ of peak areas associated with narrow and broad olefin resonances after GPC, $2 x$ PR, and 3x PR purifications, respectively. (G) Ligand populations for narrow and broad olefin resonances observed in VT-NMR for each purification method at $20^{\circ} \mathrm{C}$ as determined by comparison to ferrocene peak integral at $\delta \approx 4 \mathrm{ppm}$ (not shown). 
Figure $\mathbf{S 7}$.

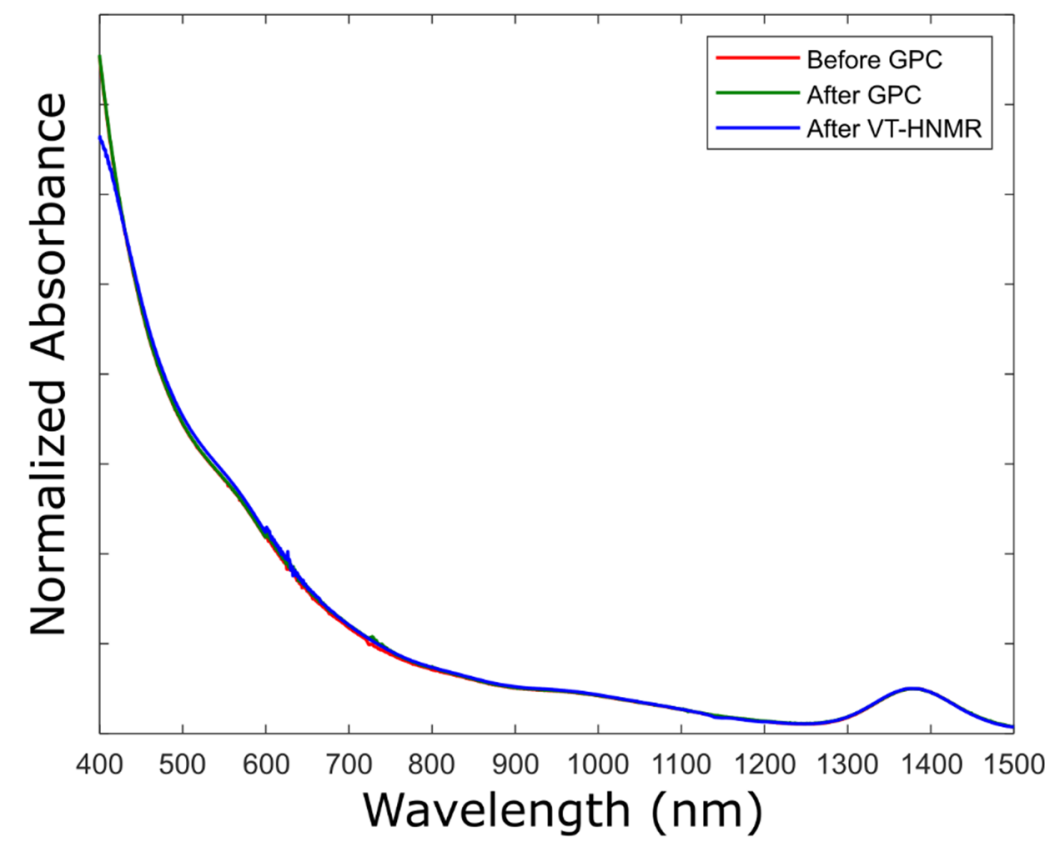

Absorbance spectra of PbS QDs before GPC, after GPC, and after VT-NMR.

Figure S8.

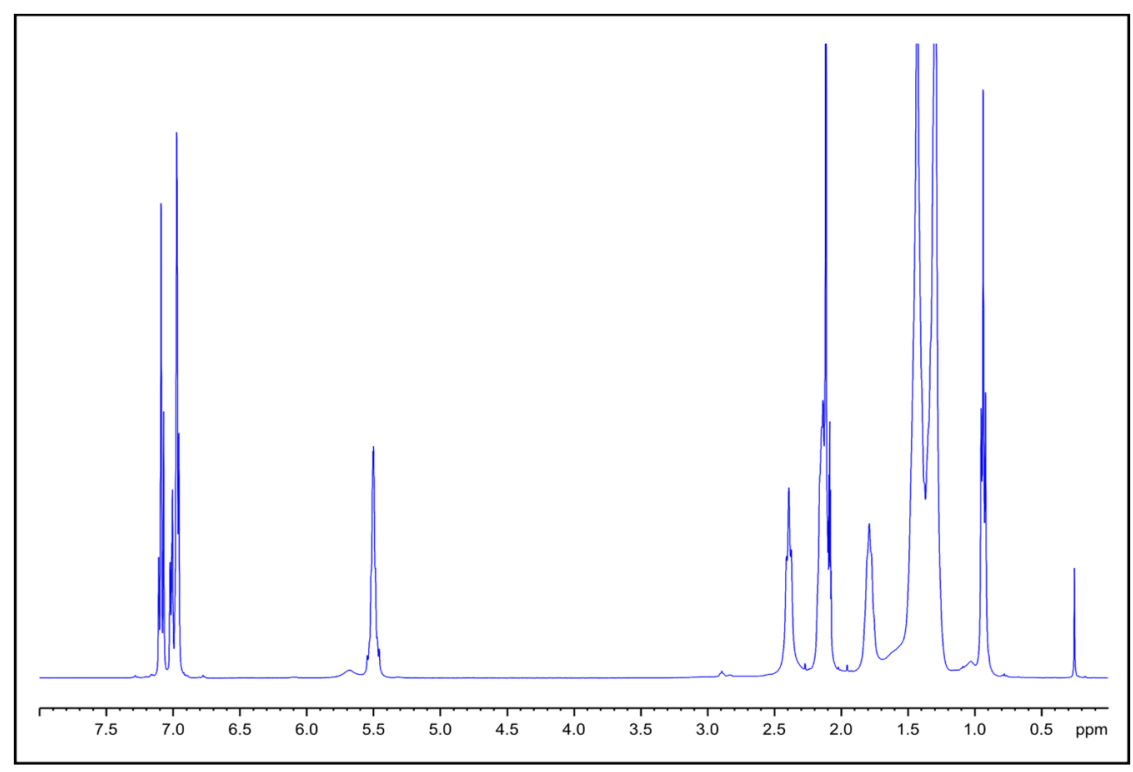

${ }^{1} \mathrm{H}$ NMR of large $\mathrm{PbS}$ QDs synthesized using anhydrous $\mathrm{Pb}(\mathrm{OA}) 2(\sim 5.2 \mathrm{~nm}$ nominal diameter: absorption spectrum shown in Figure 5 of main text). 